\title{
Correction to: Effects of di (2-ethylhexyl) phthalate and high-fat diet on lipid metabolism in rats by JAK2/STAT5
}

\author{
Yuezhu Zhang ${ }^{1} \cdot$ Liting Zhou $^{1} \cdot$ Zhaoming Zhang $^{1} \cdot$ Qi Xu $^{1} \cdot$ Xu Han $^{1} \cdot$ Yaming Zhao $^{1} \cdot$ Xinyue Song $^{1}$. \\ Tianyang Zhao ${ }^{1} \cdot$ Lin $\mathrm{Ye}^{1}$
}

Published online: 27 December 2019

(C) Springer-Verlag GmbH Germany, part of Springer Nature 2019

\section{Correction to: Environmental Science and Pollution Research} https://doi.org/10.1007/s11356-019-06599-5

We found an error in the materials and methods section. Since our team used two methods for anesthesia in rats and the anesthesia method used in this paper was 3.5\% chloral hydrate anesthesia, we mistakenly wrote the anesthetic as $3 \%$ sodium pentobarbital.

The online version of the original article can be found at https://doi.org/ 10.1007/s11356-019-06599-5

Lin Ye

jlyelin@163.com

1 Department of Occupational and Environmental Health, School of Public Health, Jilin University, 1163 Xin Min Street,

Changchun 130021, China 